\title{
The association of calf circumference and all-cause, cardiovascular and cerebrovascular mortality: results from the National Health and Nutrition Examination Surveys
}

Jia Yi Huang ${ }^{1,2}$, Lin Liu' ${ }^{2}$, Kenneth Lo ${ }^{2,3}$, Yu Ling Yu², Chao Lei Chen ${ }^{2}$, Geng Shen ${ }^{1,2}$, Bin Zhang ${ }^{1,2}$, Yu Qing Huang ${ }^{2}$, Ying Qing Feng ${ }^{1,2}$

\begin{abstract}
${ }^{1}$ School of Medicine, South China University of Technology, Guangzhou, China 2Department of Cardiology, Guangdong Cardiovascular Institute, Guangdong Provincial People's Hospital, Guangdong Academy of Medical Sciences, Guangdong Provincial Key Laboratory of Coronary Heart Disease Prevention, Guangzhou, China ${ }^{3}$ Centre for Global Cardiometabolic Health, Department of Epidemiology, Brown University, Providence, USA
\end{abstract}

Submitted: 19 December 2019; Accepted: 29 February 2020 Online publication: 28 March 2021

Arch Med Sci

DOI: https://doi.org/10.5114/aoms/118575

Copyright $\odot 2021$ Termedia \& Banach

\section{Abstract}

Introduction: Studies on the association between calf circumference (CC) and mortality have been limited. Therefore, we examined the association between CC and all-cause, cardiovascular and cerebrovascular mortality in the present study.

Material and methods: The data were retrieved from the 1999-2006 National Health and Nutritional Examination Surveys (NHANES), composed of 20,214 individuals aged $\geq 18$ years with CC being measured. We performed multivariate Cox regression models to examine the associations, then stratified the regression models into subgroups to test for interactions.

Results: Among 20,214 participants, $47.25 \%$ were men and the mean age was 45.8 years. In the fully adjusted model, each $1 \mathrm{~cm}$ increment in CC was inversely associated with the risk of all-cause mortality $(\mathrm{HR}=0.92,95 \% \mathrm{Cl}$ : $0.90-0.94, p<0.0001)$ and cardiovascular mortality $(\mathrm{HR}=0.90,95 \% \mathrm{Cl}$ : $0.84-0.97, p=0.0056$ ). Meanwhile, the highest quartile of CC had $50 \%$ $(\mathrm{HR}=0.50,95 \% \mathrm{Cl}: 0.40-0.64, p$ trend $<0.001)$ lower risk of all-cause mortality and $57 \%(\mathrm{HR}=0.43,95 \% \mathrm{Cl}: 0.21-0.88, p$ trend $=0.045)$ lower risk of cardiovascular mortality, compared to the lowest quartile of CC. For cerebrovascular mortality, CC did not have significant associations with mortality. Conclusions: Our results suggested an independently inverse association between CC and all-cause and cardiovascular mortality.

Key words: anthropometric markers, mortality, NHANES.

\section{Introduction}

Cardiovascular and cerebrovascular death remains the leading cause of mortality worldwide [1]. Plentiful factors have been reported to be associated with all-cause and cause-specific mortality, such as high-density lipoprotein cholesterol (HDL-C) [2], serum uric acid and apolipoprotein B (apoB) [3], and lower carbohydrate diets [4]. Another risk factor is the anthropometric index, which may serve as an easy-to-use indicator to iden-
Corresponding authors:

Yu Oing Huang

Department of Cardiology

Guangdong Cardiovascular

Institute

No. 106, Zhongshan Second

Road

Yuexiu District, 510080

Guangzhou, China

Phone/fax: +86-20-83827812

E-mail: hyq513@126.com

Ying Qing Feng

School of Medicine

South China University of

Technology

510632 Guangzhou, China

E-mail:651792209@qq.com 
tify the high-risk population. Calf circumference (CC) is an index reflecting lower-limb size, which represents peripheral fat and lean mass. Previous studies indicated that CC was a potential indicator to assess nutritional status and physical function, as well as an important predictor for mortality [5]. In addition, CC is easier to assess than body mass index (BMI) in frail and ill elderly patients, since the measurement of height and weight is often inaccurate or unavailable [6]. There is accumulating evidence to suggest that body composition and fat distribution are important in determining the risk of mortality, whereas global body mass may not be the best predictor [7]. Noticeably, decreased CC mainly reflects muscle loss and nutritional status. Several previous studies have found that increased size of larger limbs was associated with a reduced risk of metabolic disorder or cardiovascular disease [8].

Currently, numerous studies focus on anthropometric markers of abdominal obesity (waist circumference, waist-to-hip ratio, waist-to-height ratio) and the long-term health $[9,10]$, but it is unclear whether a marker of body composition other than abdominal obesity, namely CC, is another important indicator. To address this research gap, we have conducted this study using data from the National Health and Nutritional Examination Surveys (NHANES) population.

\section{Material and methods}

\section{Study population}

In the present study, we used publicly available data from NHANES. The study methods and procedures for data collection have been described in detail elsewhere [11]. The current study was based on the analysis of NHANES data collected from 1999 to $2006(N=41,474)$. We selected participants aged $\geq 18$ years $(n=22,624)$, and excluded participants who had missing data on calf circumference $(n=2,389)$ or mortality $(n=21)$, making a final sample of 20,214 eligible participants. The selection of the study cohort is presented in Figure 1. The ethics approval for NHANES was obtained by the Institutional Review Board of the Centers for Disease Control and Prevention.
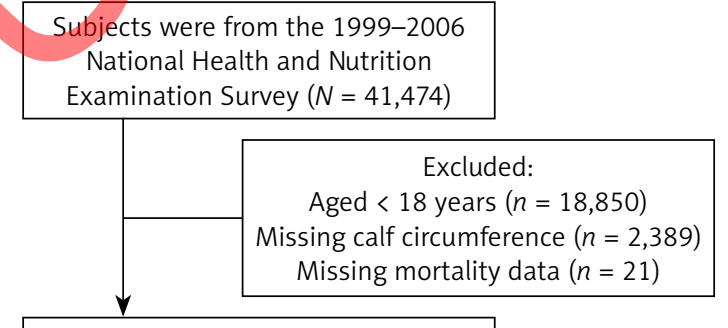

Enrolled analysis $(n=20,214)$

Figure 1. Procedure of selecting participants
Written informed consent was obtained from all participants.

\section{Data collection}

Demographic data (age, sex, race), results from physical examination and laboratory tests (systolic blood pressure (SBP), BMI, diastolic blood pressure (DBP), fasting blood glucose, total cholesterol (TC) triglyceride, HDL-C, low-density lipoprotein-cholesterol (LDL-C), estimated glomerular filtration rate (eGFR)), medical history (hypertension, diabetes, cardiovascular diseases), and prescription medication information (anti-hypertensive drugs, anti-diabetes drugs) were retrieved from the NHANES data. Cigarette smoking status was categorized as smoker, ex-smoker or non-smoker. eGFR was calculated by the Modification of Diet in Renal Disease (MDRD) formula [12]. To measure CC, technicians slid the measuring tape up and down the calf in order to find the widest point for accurate measurement. Hypertension was classified by the presence of SBP $\geq 130 \mathrm{~mm} \mathrm{Hg}$ and/or DBP $\geq 80 \mathrm{~mm} \mathrm{Hg}$, and/or currently use of anti-hypertension medication [13]. Diabetes was based on the self-reported history of diabetes or the presence of any one of the following criteria: 1) fasting glucose $\geq 126 \mathrm{mg}$ / dl; 2) non-fasting glucose $\geq 200 \mathrm{mg} / \mathrm{dl}$; 3) $\mathrm{Hb}_{\mathrm{Alc}}$ $\geq 6.5 \%$; 4) taking medication to lower blood glucose; or 5) consuming insulin [14]. Body mass index was calculated as weight in kilograms divided by squared height in meters $\left(\mathrm{kg} / \mathrm{m}^{2}\right)$. Obesity was defined as BMI $\geq 30 \mathrm{~kg} / \mathrm{m}^{2}$, overweight was defined as $B M I \geq 25 \mathrm{~kg} / \mathrm{m}^{2}$, normal weight was defined as $18.5 \mathrm{~kg} / \mathrm{m}^{2} \leq \mathrm{BMI}<25 \mathrm{~kg} / \mathrm{m}^{2}$, and underweight was defined as $\mathrm{BMI}<18.5 \mathrm{~kg} / \mathrm{m}^{2}$ [15].

\section{Outcome}

The anonymized data of NHANES 1999-2006 participants were linked to longitudinal medicare and mortality data. Data on survival status were collected from the date of survey participation until 31 December 2015. Outcomes of our study were all-cause mortality, mortality due to cardiovascular diseases (100-109, I11, I13, I20-151), and cerebrovascular diseases (160-169). The cause of death was determined using the 10th revision of the International Classification of Diseases (ICD-10).

\section{Statistical analysis}

Continuous variables were expressed as mean \pm standard deviation (SD), whereas categorical variables were presented as numbers with corresponding percentages. CC was divided into quartiles (Q1-Q4). Baseline characteristics were summarized based on CC quartiles. The Kruskal-Wallis $H$, oneway ANOVA and $\chi^{2}$ tests were applied to determine whether the subgroup difference was significant. 
Multivariate Cox regression was performed to assess the association between CC and the risk of all-cause, cardiovascular and cerebrovascular mortality. CC was treated as both continuous (per $1 \mathrm{~cm}$ increment) and categorical variables (Q1-Q4) and was put into separate models. We computed three regression models which were adjusted for different sets of confounders. The crude model only included CC. Model 1 was further adjusted for age, gender and BMI. Meanwhile, Model 2 was adjusted for age, gender, BMI, SBP, TC, HDL-C, use of antihypertensive drugs and anti-diabetic drugs, eGFR, alcohol drinking, serum albumin, smoking status, race; history of diabetes, hypertension or cardiovascular diseases. The results were reported as the hazard ratio (HR) with 95\% confidence interval $(\mathrm{Cl})$.

To test the robustness of our results, we further stratified the regression models into subgroups and tested for the interaction. The cohort was divided into groups based on gender, age, hypertension diabetes, BMI, SBP and smoking status. When analysis a subgroup variable, age, gender, BMI, SBP, TC, HDL-C, use of antihypertensive drugs and anti-diabetic drugs, eGFR, alcohol drinking, smoking status, race, history diabetes, hypertension or cardiovascular diseases were all adjusted except the variable itself. The Kaplan-Meier analysis was conducted to compare the difference in survival rates by CC quartiles, and the intergroup differences were estimated by the log-rank test. Cubic spline models were used to estimate the HRs for mortality associated with increasing CC. A two-sided $p$ value $<0.05$ was consid ered statistically significant. Data management and analyses were performed using the statistical software package R version 3.32 (http://www.R-project. org, The R Foundation, Vienna, Austria).

\section{Results}

\section{Baseline characteristics}

Table I summarizes the characteristics of participants based on CC quartiles. Overall, 20,214 participants were included, with a mean age of 45.8 years; $47.75 \%$ were men. During the follow-up period of 11.82 years, 3655 deaths were recorded, including 466 cardiovascular disease deaths and 152 cerebrovascular disease deaths. Participants with the highest quartile of CC had the lowest proportion of all-cause (11.25\%), cardiovascular (1.36\%) and cerebrovascular mortality $(0.37 \%)$. All variables were significantly different by quartiles (all $p<0.05$ ).

Relationships between CC and all-cause, cardiovascular and cerebrovascular disease mortality

Results from Cox regression models are shown in Table II. When CC was expressed as a contin- uous variable, each $1 \mathrm{~cm}$ increment of CC was inversely associated with all-cause mortality. The corresponding HR was 0.90 (95\% Cl: 0.90-0.91, $p<0.0001)$ in the crude model, $0.91(95 \% \mathrm{Cl}$ : 0.90-0.92, $p<0.0001$ ) in Model 1 and increased CC was still significantly associated with lower risk of all-cause mortality in Model $2(\mathrm{HR}=0.92$, 95\% Cl: 0.90-0.94, $p<0.0001)$. Regarding cardiovascular death, it showed a similar pattern; CC was significantly associated with cardiovascular mortality in Model $2(\mathrm{HR}=0.90,95 \% \mathrm{Cl}$ : $0.84-0.97, p=0.0056)$. For cerebrovascular death, a significant association was found in the crude model and Model 1, but not Model 2.

When CC was expressed as a categorical variable, the risk of all-cause mortality was significantly lower for participants with the highest quartile of CC in Model 2 ( $\mathrm{HR}=0.50,95 \% \mathrm{Cl}: 0.40-0.64$, $p$ trend $<0.001$ ) when using the lowest quartile as the referent. Similarly, the highest quartile of CC was significantly associated with a lower risk of cardiovascular mortality in Model 2 (Q4: HR = $0.43,95 \% \mathrm{Cl}: 0.21-0.88, p$ trend $=0.045)$. The calf circumference was not associated with cerebrovascular mortality when treated as a categorical variable.

In Figure 2, the Kaplan-Meier survival curves plotting quartiles of CC showed that the rates of all-cause and cardiovascular death were statistically significantly different by CC quartiles (both log rank: $p<0.001$ ). Figure 3 demonstrates the $\mathrm{cu}$ bic spline models estimating the HRs for all-cause and cause-specific mortality associated with increasing CC.

\section{Subgroup analysis}

Table III summarizes the association between CC and mortality in subgroups. Among all the subgroups, only BMI significantly modified the associations between CC and mortality risk. The HR of all-cause mortality was $0.80(95 \% \mathrm{Cl}: 0.79-0.82)$ in the subgroup with $\mathrm{BMI}<25 \mathrm{~kg} / \mathrm{m}^{2}$, which was significantly lower than in subjects with $\mathrm{BMI} \geq$ $25 \mathrm{~kg} / \mathrm{m}^{2}(\mathrm{HR}=0.88$; 95\% Cl: 0.87-0.89) ( $p$ for interaction $<0.0001)$. A similar pattern was also found for the association between CC and cardiovascular mortality. For the subgroup with $\mathrm{BMI}$ $<25 \mathrm{~kg} / \mathrm{m}^{2}$, the HR was 0.74 (95\% Cl: 0.70-0.78) and when $\mathrm{BMI} \geq 25 \mathrm{~kg} / \mathrm{m}^{2}$, the HR was $0.88(95 \%$ Cl: 0.85-0.91) ( $p$ for interaction $=0.0005)$.

\section{Discussion}

The current study investigated the association between CC and all-cause, cardiovascular and cerebrovascular mortality. We found that CC was inversely associated with all-cause and cardiovascular mortality. For every $1 \mathrm{~cm}$ increment in CC, 


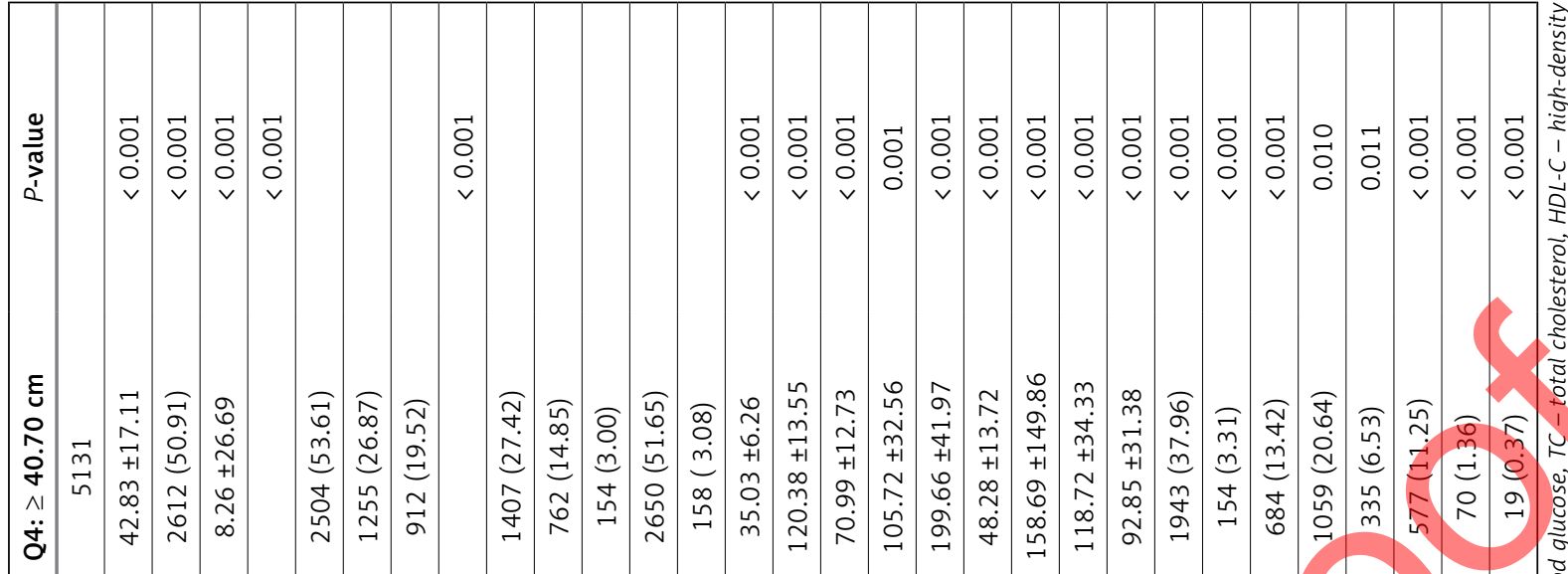

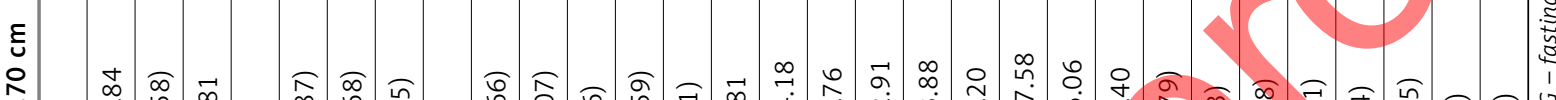

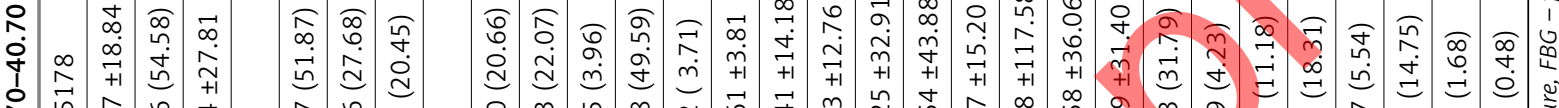

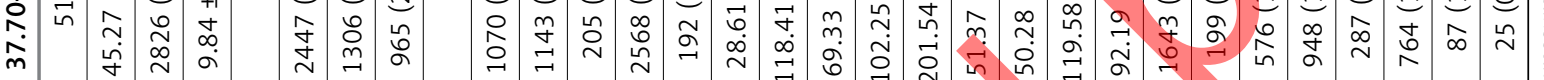
$\ddot{\tilde{O}}$

ร

$\therefore$ 일

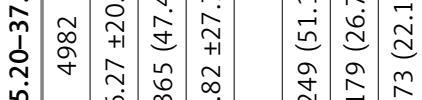

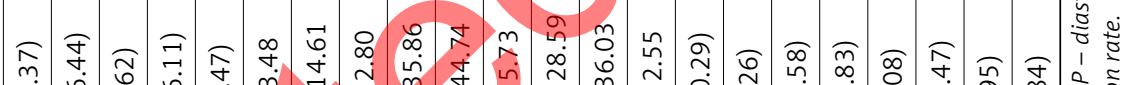

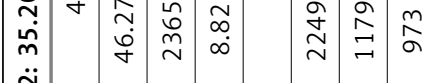

$\ddot{\check{\sigma}}$

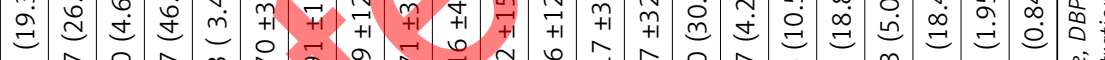

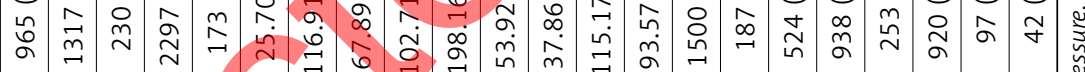

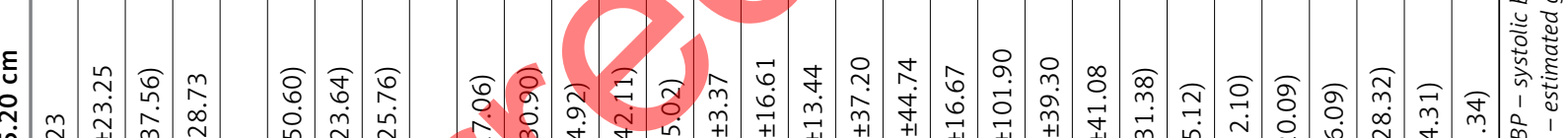

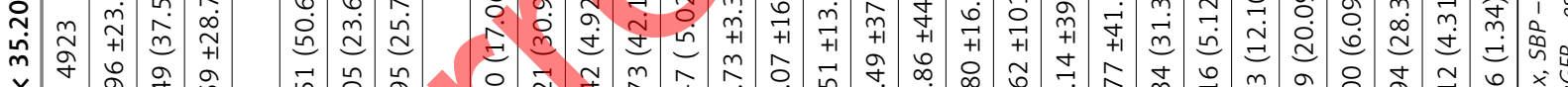

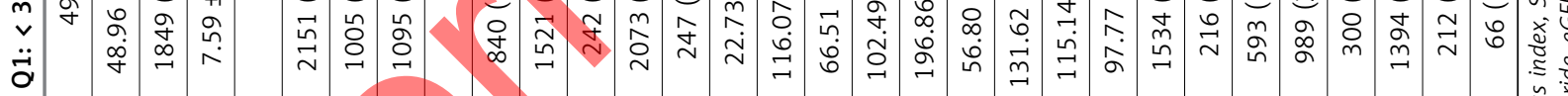

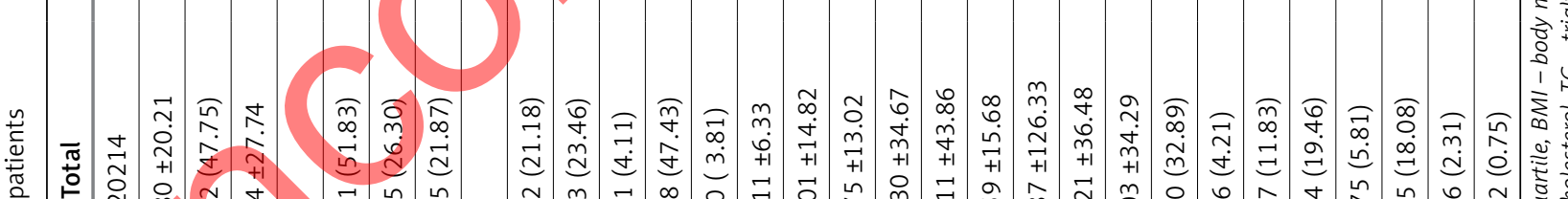

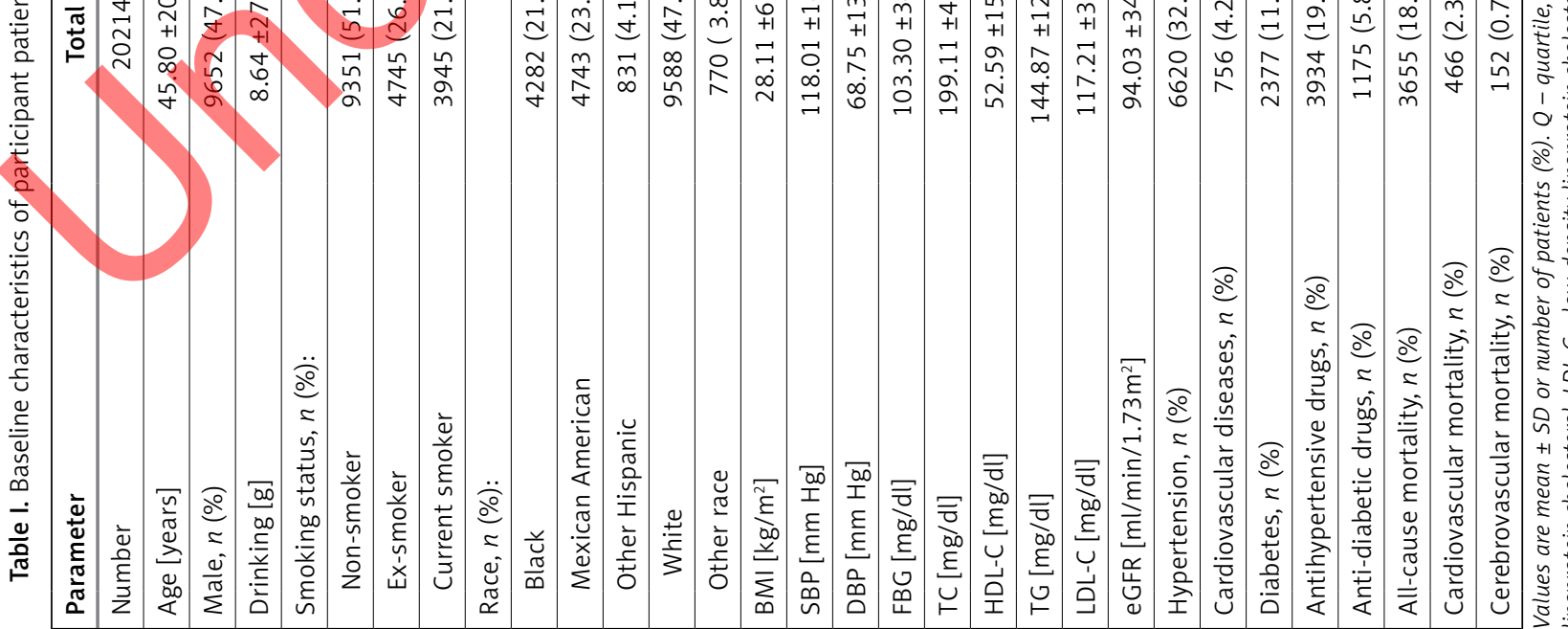
है ญे ㄴ. 8
0
0
0
0

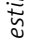


The association of calf circumference and all-cause, cardiovascular and cerebrovascular mortality: results from the National Health and Nutrition Examination Surveys

Table II. Cox regression analysis for all-cause, cardiovascular and cerebrovascular disease mortality

\begin{tabular}{|c|c|c|c|}
\hline Exposure & $\begin{array}{c}\text { Non-adjusted } \\
\text { HR }(95 \% \mathrm{Cl}), p \text {-value }\end{array}$ & $\begin{array}{c}\text { Model } 1 \\
\text { HR }(95 \% \mathrm{Cl}), p \text {-value }\end{array}$ & $\begin{array}{c}\text { Model } 2 \\
\text { HR }(95 \% \mathrm{Cl}), p \text {-value }\end{array}$ \\
\hline \multicolumn{4}{|l|}{ All-cause mortality } \\
\hline $\begin{array}{l}\text { Calf circumference } \\
\text { (per } 1 \mathrm{~cm} \text { increment) }\end{array}$ & $0.90(0.90,0.91),<0.0001$ & $0.91(0.90,0.92),<0.0001$ & $0.92(0.90,0.94),<0.0001$ \\
\hline \multicolumn{4}{|c|}{ Calf circumference groups: } \\
\hline Q1 & 1.0 & 1.0 & 1.0 \\
\hline Q2 & $0.61(0.56,0.67),<0.0001$ & $0.66(0.61,0.73),<0.0001$ & $0.70(0.60,0.81),<0.0001$ \\
\hline Q3 & $0.48(0.44,0.53),<0.0001$ & $0.57(0.51,0.63),<0.0001$ & $0.64(0.54,0.75),<0.0001$ \\
\hline Q4 & $0.36(0.33,0.40),<0.0001$ & $0.49(0.43,0.57),<0.0001$ & $0.50(0.40,0.64),<0.0001$ \\
\hline$P$ for trend & $<0.001$ & $<0.001$ & $<0.001$ \\
\hline \multicolumn{4}{|c|}{ Cardiovascular mortality } \\
\hline $\begin{array}{l}\text { Calf circumference } \\
\text { (per } 1 \mathrm{~cm} \text { increment) }\end{array}$ & $0.88(0.86,0.90),<0.0001$ & $0.87(0.84,0.91),<0.0001$ & $4,0.97), 0.0056$ \\
\hline \multicolumn{4}{|c|}{ Calf circumference groups: } \\
\hline Q1 & 1.0 & 1.0 & \\
\hline Q2 & $0.43(0.34,0.54),<0.0001$ & $0.48(0.37,0.62),<0.0001$ & $0.34(0.21,0.56),<0.0001$ \\
\hline Q3 & $0.36(0.28,0.47),<0.0001$ & $0.44(0.33,0.60),<0.0001$ & $0.38(0.22,0.64), 0.0003$ \\
\hline Q4 & $0.29(0.22,0.38),<0.0001$ & $0.41(0.28,0.62),<0.0001$ & $0.43(0.21,0.88), 0.0205$ \\
\hline$P$ for trend & $<0.001$ & $<0.001$ & 0.045 \\
\hline \multicolumn{4}{|c|}{ Cerebrovascular mortality } \\
\hline $\begin{array}{l}\text { Calf circumference } \\
\text { (per } 1 \mathrm{~cm} \text { increment) }\end{array}$ & $0.86(0.83,0.90),<0.0001$ & $84,0.96), 0.0028$ & 0.91 (0.80, 1.03), 0.1201 \\
\hline \multicolumn{4}{|c|}{ Calf circumference groups: } \\
\hline Q1 & 1.0 & 1.0 & 1.0 \\
\hline Q2 & $0.59(0.40,0.88), 0.0085$ & $0.76(0.49,1.17), 0.2118$ & $0.64(0.28,1.46), 0.2893$ \\
\hline Q3 & $0.34(0.21,0.53),<0.0001$ & $0.55(0.32,0.94), 0.0293$ & $0.89(0.37,2.16), 0.8052$ \\
\hline Q4 & $0.25(0.15,0.42),<0.0001$ & $0.52(0.25,1.10), 0.0862$ & $0.70(0.19,2.56), 0.5943$ \\
\hline$P$ for trend & $\wedge<0.001$ & 0.377 & 0.602 \\
\hline
\end{tabular}

Non-adjusted adjusted for none. Model 1 was adjusted for age, gender and BMI. Model 2 was adjusted for age, gender, BMI, SBP, TC, HDL-C, antihypertensive drugs, anti-diabetic drugs, eGFR, drinking, serum albumin, smoking status, race, diabetes, hypertension, cardiovascular diseases. $Q$ - quartile, $H R$ - hazard ratio, $\mathrm{Cl}$ - confidence interval.

there was an $8 \%$ reduction in the risk of all-cause mortality and a $10 \%$ reduction for cardiovascular mortality. For subjects with the highest quartile of CC $(\geq 40.70 \mathrm{~cm})$, there was a $50 \%$ reduction in the risk of all-cause mortality, and $57 \%$ reduction in the risk of cardiovascular mortality.

The calf circumference is an indicator that is simple, non-invasive and easy to obtain in routine clinical practice. However, evaluation of the potential of CC to predict mortality is limited. Previous studies have revealed that CC was closely related to lean muscle mass [16], but the relationship between CC and cause-specific mortality has not been investigated in detail. Prior studies mainly investigated CC among elderly and fragile patients, and found that low CC was suggested to be an indicator of malnutrition in the elderly population $[17,18]$. In contrast, a study reported that CC was not an independent predictor of mortality risk after adjusting for other prognostic factors in older patients with cardiovascular disease [19].
The present study focused on the general population and included relatively young and healthy individuals. The results of this study suggested that the usage of CC might be applied to a larger population scale.

Regarding the subgroup analysis, the relationships between CC and mortality differed according to BMI. The calf circumference had a stronger inverse association with mortality among participants with $\mathrm{BMI}<25 \mathrm{~kg} / \mathrm{m}^{2}$. This is consistent with the well-established theory that BMI is an independent risk factor for mortality [20], which might weaken the protective effects of increased CC. Also, a study conducted among 160 patients aged $\geq 65$ years revealed CC to be a better indicator than BMI in predicting mortality [21]. The calf circumference is potentially a protective factor for mortality, particular for those with lower BMI.

The underlying mechanism of CC relating to allcause and cardiovascular mortality has not been fully investigated, but several potential mecha- 


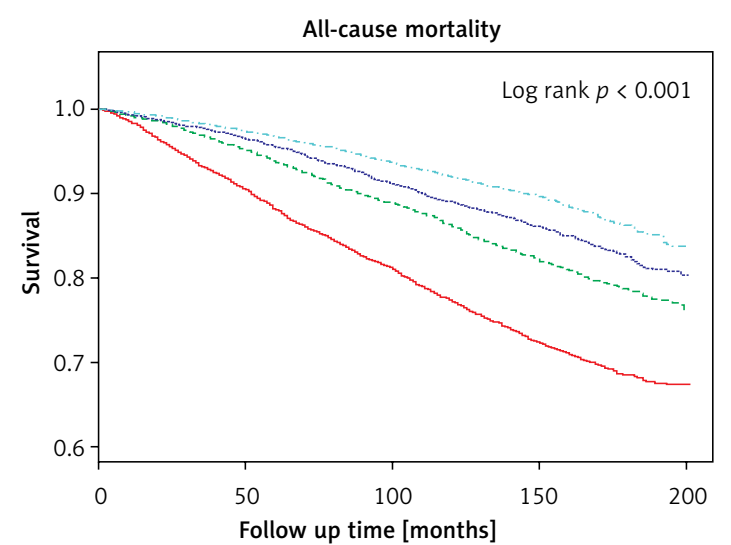

Maximal calf circumference groups [cm]

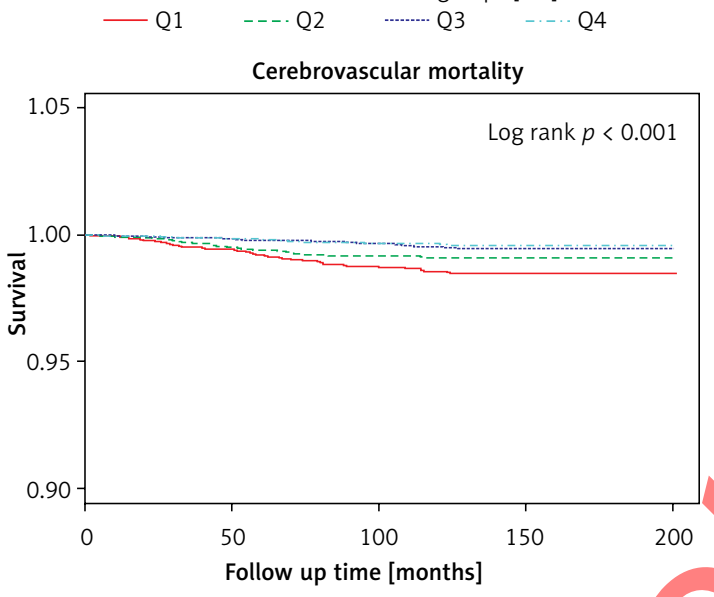

Maximal calf circumference groups [cm] - Q1 - -.. Q2 …-... Q3
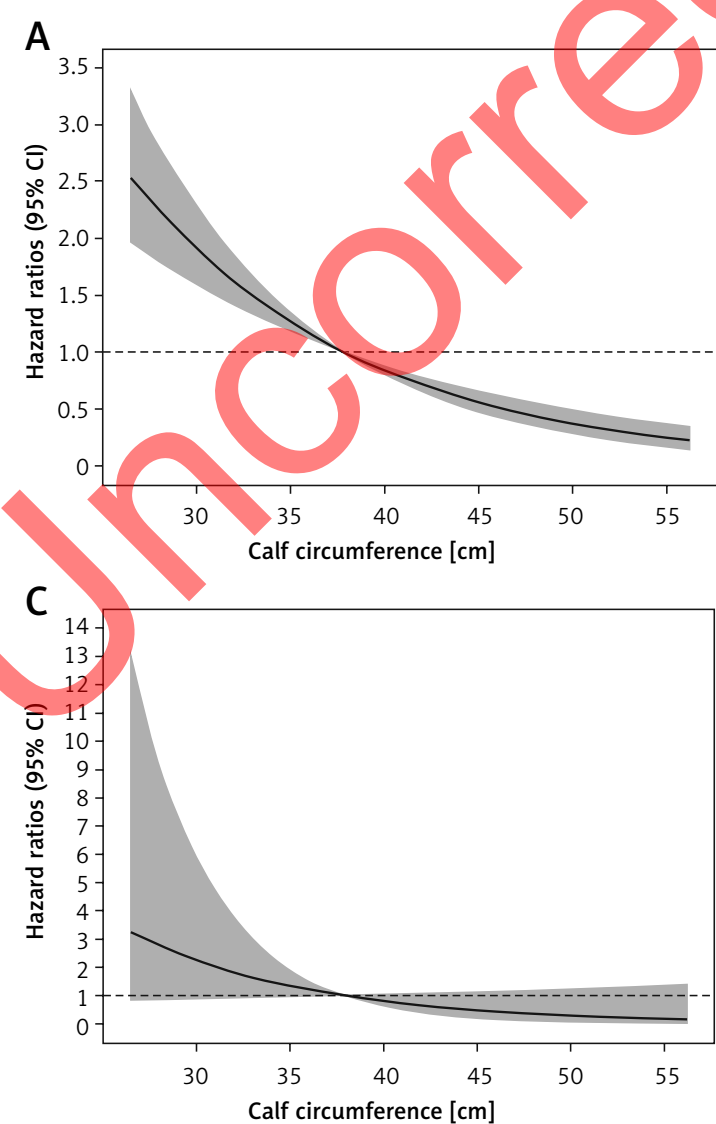

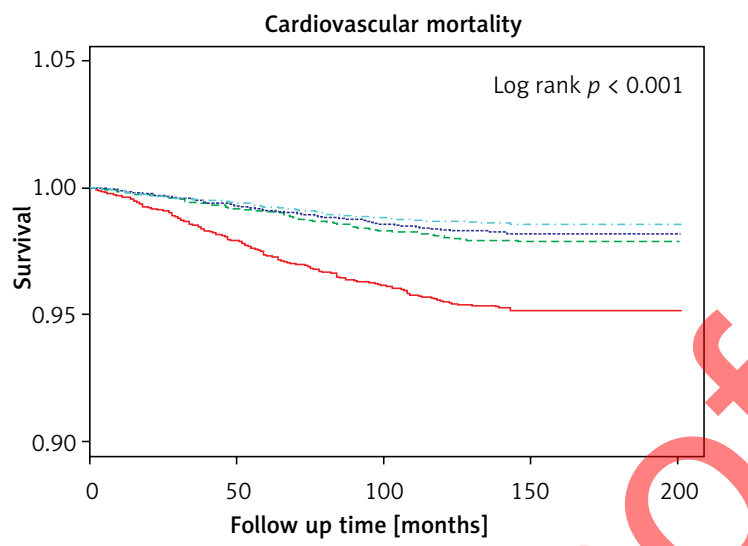

Maximal calf circumference groups [cm]
Figure 2. The Kaplan-Meier survival curves plotting quartiles of calf circumference

$Q$ - quartile.
B

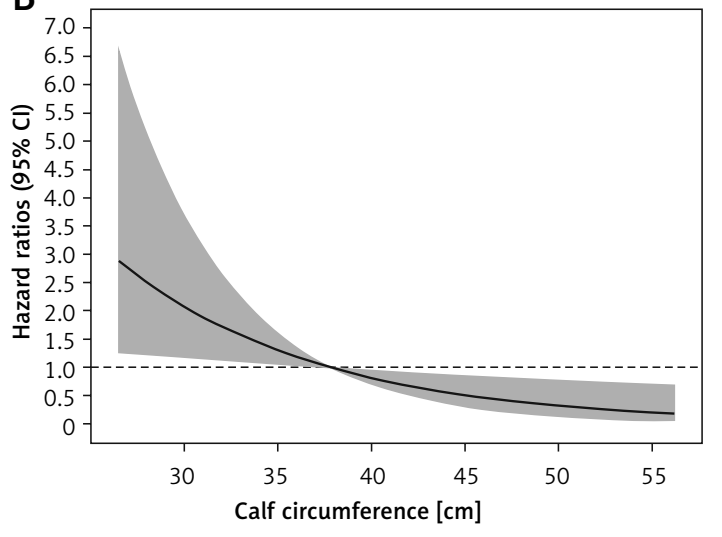

Figure 3. Cubic spline of hazard ratios for all-cause and cause specific mortality associated with increasing calf circumference. A - Association of calf circumference $(\mathrm{cm})$ and hazard ratios of all-cause mortality. B - Association of calf circumference $(\mathrm{cm})$ and hazard ratios of cardiovascular mortality. $\mathrm{C}$ - Association of calf circumference $(\mathrm{cm})$ and hazard ratios of cerebrovascular mortality. Age, gender, BMI, SBP, TC, HDL-C, antihypertensive drugs, anti-diabetic drugs, eGFR, serum albumin, alcohol drinking, smoking status, race, diabetes, hypertension, cardiovascular diseases were all adjusted 
Table III. Subgroup analysis for calf circumference with all-cause and cardiovascular mortality

\begin{tabular}{|c|c|c|c|c|c|}
\hline \multirow[t]{2}{*}{ Parameter } & \multicolumn{2}{|r|}{ All-cause mortality } & \multicolumn{3}{|c|}{ Cardiovascular mortality } \\
\hline & Number & HR (95\% Cl), $p$-value & $P$ interaction & HR (95\% Cl), $p$-value & $P$ interaction \\
\hline Gender: & & & 0.1219 & & 0.0829 \\
\hline Male & 9652 & $0.89(0.88,0.90),<0.0001$ & & $0.87(0.84,0.90),<0.0001$ & \\
\hline Female & 10562 & $0.90(0.89,0.91),<0.0001$ & & $0.86(0.83,0.89),<0.0001$ & \\
\hline Age: & & & 0.6140 & & 0.5837 \\
\hline$<50$ & 11856 & $0.96(0.94,0.98),<0.0001$ & & $0.99(0.92,1.08), 0.8686$ & \\
\hline$\geq 50$ & 8358 & $0.91(0.90,0.92),<0.0001$ & & $0.89(0.87,0.91),<0.0001$ & \\
\hline Diabetes: & & & 0.2294 & & 573 \\
\hline No & 17978 & $0.89(0.88,0.90),<0.0001$ & & $0.88(0.85,0.90),<0.0001$ & \\
\hline Yes & 2080 & $0.92(0.91,0.94),<0.0001$ & & $0.88(0.84,0.92),<0.0001$ & \\
\hline Hypertension: & & & 0.0769 & & 0.1296 \\
\hline No & 14210 & $0.88(0.87,0.90),<0.0001$ & & $0.79(0.76,0.82)$ & \\
\hline Yes & 5914 & $0.90(0.89,0.91),<0.0001$ & & $0.90(0.88,0.93),<0.0001$ & \\
\hline BMI: & & & $<0.0001$ & & 0.0005 \\
\hline$<25$ & 6888 & $0.80(0.79,0.82),<0.0001$ & & $0.74(0.70,0.78),<0.0001$ & \\
\hline$\geq 25$ & 13122 & $0.88(0.87,0.89),<0.0001$ & & $0.88(0.85,0.91),<0.0001$ & \\
\hline SBP: & & & 0.8037 & & 0.6892 \\
\hline$<140$ & 15470 & $0.92(0.91,0.93),<0.0001$ & & $0.89(0.86,0.92),<0.0001$ & \\
\hline$\geq 140$ & 612 & $0.90(0.86,0.93),<0.0001$ & & $0.86(0.78,0.94), 0.0012$ & \\
\hline Smoking: & & & & & 0.1757 \\
\hline Non-smoker & 9351 & $0.90(0.88,0.91),<0.0001$ & & $0.88(0.85,0.91),<0.0001$ & \\
\hline Ex-smoker & 4745 & $0.89(0.87,0.90),<0.0001$ & & $0.85(0.82,0.89),<0.0001$ & \\
\hline Current smoker & 3945 & $0.90(0.88,0.92),<0.0001$ & & $0.87(0.82,0.92),<0.0001$ & \\
\hline
\end{tabular}

When analyzing a subgroup variable, age, gender, BMI, SBP, TC, HDL-C, antihypertensive drugs, anti-diabetic drugs, eGFR, serum albumin, drinking, smoking status, race, diabetes, hypertension, cardiovascular diseases were all adjusted except the variable itself. HR - hazard ratio, $\mathrm{Cl}$-confidence interval, $\mathrm{BMI}$ - body mass index, SBP - systolic blood pressure.

nisms have been proposed. The calf circumference is a marker of peripheral subcutaneous fat, besides lean mass [22]. Several studies have suggested that the association of adiposity distribution with mortality might be partially explained by inflammation [23]. A study revealed that a larger leg fat mass was related to lower fasting and postprandial glucose levels [8]. Some other previous studies showed that a greater CC might have an antiatherogenic effect $[24,25]$. For example, a larger CC reduces the frequency of carotid plaques, which give a plausible reason for a lower risk for cardiovascular mortality [25]. A few studies suggested that peripheral fat mass could protect against unstable glucose and lipid metabolites, which in turn reduce adverse effect of fat on cardiometabolic risk [26]. Some other studies implied that polymorphisms were associated with hypertension and, consequently, with a higher risk of cardiovascular mortality [27]. Similarly, CC might also be affected by genetic composition. These results may explain the protective role of a greater CC in all-cause and cardiovascular mortality.

Further prospective studies are expected to elucidate the link between CC and mortality among the general population with various demographics, and to reveal the underlying mechanism between CC and mortality. There were several limitations of our study. First, the CC values varied according to whether the participant was standing or lying. Second, we did not obtain the status of chronic heart failure and chronic kidney disease. Third, some data were self-reported and might be subject to recall bias. Fourth, more sophisticated anthropometric measurements such as dual energy X-ray absorptiometry were not available in this study. Fifth, the optimal cut-off values of CC were calculated from the NHANES data, which mainly represent US citizens. Replication studies are required in an external population.

In conclusion, CC was inversely associated with the risk of all-cause and cardiovascular mortality. CC had a more significant protective effect on those with $\mathrm{BMI}<25 \mathrm{~kg} / \mathrm{m}^{2}$.

\section{Acknowledgements}

Jia Yi Huang and Lin Liu are equal authors of this paper.

During the process of data analysis, writing and submission of the article, this work was supported 
by the Science and Technology Program of Guangzhou (No. 201604020143, No. 201604020018, No. 201604020186, No. 201510010254, and No. 201803040012), and the National Key Research and Development Program of China (No. 2017FYC1307603, No. 2016YFC1301305, and No. 2017YFC0909303) and the Key Area R\&D Program of Guangdong Province (No. 2019B020227005).

\section{Conflict of interest}

The authors declare no conflict of interest.

\section{References}

1. GBD 2017 Causes of Death Collaborators. Global, regional, and national age-sex-specific mortality for 282 causes of death in 195 countries and territories, 19802017: a systematic analysis for the Global Burden of Disease Study 2017. Lancet 2018; 392: 1736-88.

2. Mazidi M, Mikhailidis DP, Banach M. Associations between risk of overall mortality, cause-specific mortality and level of inflammatory factors with extremely low and high high-density lipoprotein cholesterol levels among American adults. Int J Cardiol 2019; 276: 242-7.

3. Mazidi M, Katsiki N, Mikhailidis DP, Bartlomiejczyk MA Banach M. Association of empirical dietary atherogenic indices with all-cause and cause-specific mortality in a multi-ethnic adult population of the United States. Nutrients 2019; 11: 2323.

4. Mazidi M, Katsiki N, Mikhailidis DP, Sattar N, Banach M. Lower carbohydrate diets and all-cause and cause-specific mortality: a population-based cohort study and pooling of prospective studies. Eur Heart J 2019; 40: 2870-9.

5. Lin SJ, Hwang SJ, Liu CY, Lin HR. The relationship between nutritional status and physical function, admission frequency, length of hospital stay, and mortality in old people living in long-term care facilities. J Nurs Res 2012; 20; 110-21.

6. Weng $\mathrm{CH}$, Tien $\mathrm{CP}$, $\mathrm{Li} \mathrm{Cl}$, et al. Mid-upper arm circumference, calf circumference and mortality in Chinese longterm care facility residents: a prospective cohort study. BMJ Open 2018; 8: e20485.

7. Konieczna J, Abete I, Galmes AM, et al. Body adiposity indicators and cardiometabolic risk: Cross-sectional analysis in participants from the PREDIMED-Plus trial. Clin Nutr 2019; 38: 1883-91.

8. Snijder MB, Dekker JM, Visser M, et al. Trunk fat and leg fat have independent and opposite associations with fasting and postload glucose levels: the Hoorn study. Diabetes Care 2004; 27: 372-7.

9. Lo K, Liu Q, Allison M, et al. Prospective associations of waist-to-height ratio with cardiovascular events in postmenopausal women: results from the Women's Health Initiative. Diabetes Care 2019; 9: e148-9.

10. Lo K, Wong M, Khalechelvam P, Tam W. Waist-to-height ratio, body mass index and waist circumference for screening paediatric cardio-metabolic risk factors: a meta-analysis. Obes Rev 2016; 12: 1258-75.

11. CDC, Centers For Disease Control. About the National Health and Nutrition Examination Survey. 2017. https:// www.cdc.gov/nchs/nhanes/about_nhanes.htm.

12. Levey AS, Stevens $L A$, Schmid $C H$, et al. A new equation to estimate glomerular filtration rate. Ann Intern Med 2009; 150: 604-12.
13. Whelton PK, Carey RM, Aronow WS, et al. 2017 ACC/ AHA/AAPA/ABC/ACPM/AGS/APhA/ASH/ASPC/NMA/ PCNA Guideline for the Prevention, Detection, Evaluation, and Management of High Blood Pressure in Adults: A Report of the American College of Cardiology/American Heart Association Task Force on Clinical Practice Guidelines. Circulation 2018; 138: e426-83.

14. Association of American Diabetes. Standards of medical care for patients with diabetes mellitus. Diabetes Care 2003; 26 Suppl. 1: S33-50.

15. Jensen MD, Ryan DH, Apovian CM, et al. 2013 AHA/ACC/ TOS Guideline for the Management of Overweight and Obesity in Adults. Circulation 2014; 129 (25 suppl. 2): S102-38.

16. Tsai AC, Chang TL. The effectiveness of BMI, calf circumference and mid-arm circumference in predicting subsequent mortality risk in elderly Taiwanese. Br $>\mathrm{Nutr}$ 2011; 105: 275-81.

17. Bonnefoy M, Jauffret M, Kostka T, Jusot JF. Usefulness of calf circumference measurement in assessing the nutritional state of hospitalized elderly people. Gerontology 2002; 48: 162-9.

18. Kaiser MJ, Bauer JM, Ramsch C, et al. Validation of the Mini Nutritional Assessment short-form (MNA-SF): a practical tool for identification of nutritional status. J Nutr Health Aging 2009; 13: 782-8.

19. Kamiya K, Masuda T, Matsue Y, et al. Prognostic usefulness of arm and calf circumference in patients $\geq 65$ years of age with cardiovascular disease. Am J Cardiol 2017; 119: 186-91.

20. Chen Z, Yang G, Offer A, et al. Body mass index and mortality in China: a 15-year prospective study of 220000 men. Int J Epidemiol 2012; 41: 472-81.

21. Tsai AC, Lai MC, Chang TL. Mid-arm and calf circumferences (MAC and CC) are better than body mass index (BMI) in predicting health status and mortality risk in institutionalized elderly Taiwanese. Arch Gerontol Geriatr 2012; 54: 443-7.

22. Rolland Y, Lauwers-Cances V, Cournot M, et al. Sarcopenia, calf circumference, and physical function of elderly women: a cross-sectional study. J Am Geriatr Soc 2003; 51: 1120-4.

23. Livshits G, Kalinkovich A. Inflammaging as a common ground for the development and maintenance of sarcopenia, obesity, cardiomyopathy and dysbiosis. Ageing Res Rev 2019; 56: 100980.

24. Tanko LB, Bagger YZ, Alexandersen P, Larsen PJ, Christiansen C. Peripheral adiposity exhibits an independent dominant antiatherogenic effect in elderly women. Circulation 2003; 107: 1626-31.

25. Debette S, Leone N, Courbon D, et al. Calf circumference is inversely associated with carotid plaques. Stroke 2008; 39: 2958-65.

26. Peppa M, Koliaki C, Hadjidakis DI, et al. Regional fat distribution and cardiometabolic risk in healthy postmenopausal women. Eur J Intern Med 2013; 24: 824-31.

27. Neto A, Farias M, Vasconcelos N, Xavier Jr AF, Assunção $M L$, Ferreira HS. Prevalence of endothelial nitric oxide synthase (ENOS) gene G894T polymorphism and its association with hypertension: a population-based study with Brazilian women. Arch Med Sci Atheroscler Dis 2019; 18: e63-73. 